\title{
The Reconstruction of Popliteal Artery Injury after Knee Dislocation by Catheter-Directed Thrombolysis
}

\section{Chunshui He ${ }^{1 *}$, Yong $\mathrm{Hu}^{2}$, Yong Huang ${ }^{3}$, Xiaohong Fan ${ }^{3}$, Yuan Liu and Yang Liu ${ }^{1}$}

${ }^{1}$ Department of vascular Surgery, University Hospital of Chengdu University of Traditional Chinese Medicine, No. 39 Shier Qiao Road, Chengdu 610071, China

${ }^{2}$ Department of Joint, Sichuan Orthopedic Hospital, Chengdu 610071, China

${ }^{3}$ Department of Orthopedic, University Hospital of Chengdu University of Traditional Chinese Medicine, Chengdu 610071, China

\section{Introduction}

Popliteal artery injury is uncommon after knee dislocation, and the surgical revascularization is still the standard treatment. We present a 19 years old male patient who sustained a trauma to the right knee resulting in posterior dislocation of knee joint and blunt popliteal artery injury had an endovascular repair by catheter-directed thrombolysis successfully. Although a few papers have described the endovascular treatment of blunt popliteal artery injury by implanting the stents in the popliteal artery before, this case is an exploration to solve this kind of lesion without leaving stent behind to avoid the possibility of stent related complications.

Knee dislocation itself is an infrequent injury and it accounts for less than $1 \%$ of all extremity injuries, and the prevalence of vascular injury associated with knee dislocation is about 30\% [1]. The standard vascular repair when encountering a blunt damage of popliteal artery is through an interposition saphenous vein graft, although a prolonged period of postoperative recovery is common $[1,2]$. Minimal invasive endovascular therapies offer an attractive treatment alternative due to the ability to perform diagnostic evaluation and therapeutic intervention nearly simultaneously with a percutaneous approach. In addition, faster access to difficult anatomical locations is usually possible, resulting in less blood loss and shorter recovery time. A few papers have described the reconstruction of the damaged popliteal artery by stents implanting [3,4] although the site of popliteal artery is not suitable for stent implantation since the high frequency of stent fracture. Basing on the pathological change of

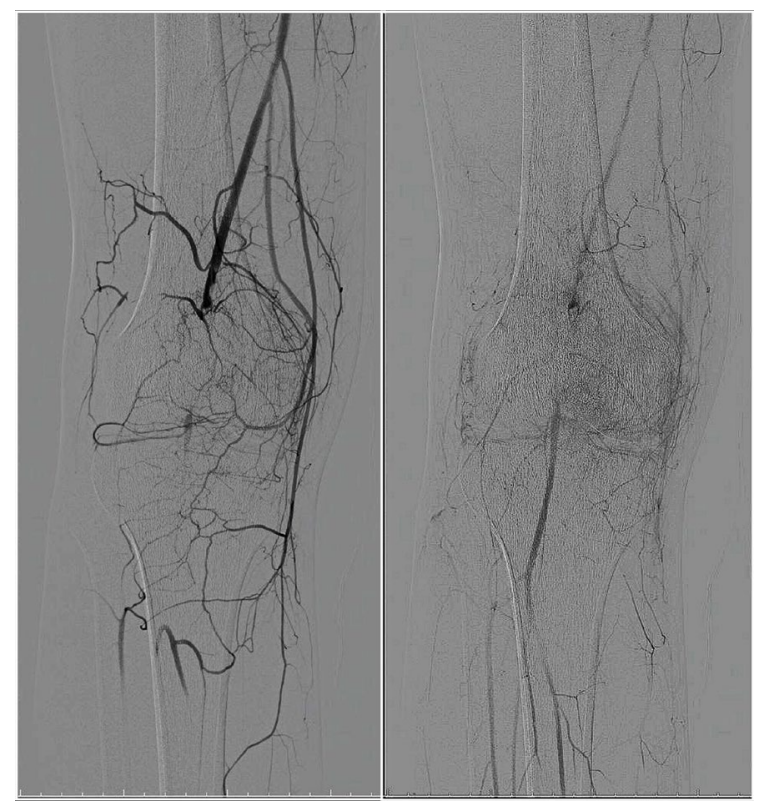

Figure 1: Popliteal artery injury and thrombus. popliteal artery injury after knee dislocation is the damage of vessel wall and the development of acute thrombosis subsequently; we want to solve this kind of lesion by catheter-directed thrombolysis without leaving anything behind.

\section{Case Report}

A 19-year-old male transferred from an orthopedic hospital to our department after a heavy stuff fallen on his right knee causing the posterior dislocation, blunt injury to the popliteal artery and no nerve damage 14 days after the trauma. He sustained no open wound with obvious deformity of his right knee with rest pain, low temperature of right foot, and non palpable pulses. The MRI of knee demonstrated the rupture of both anterior and posterior cruciate ligament, and the ankle-brachial index (ABI) test showed that the right was 0.46 and the left was 0.92 , and the ultrasound indicated the right popliteal artery occlusion. The patient was not on any anticoagulation therapy from injury to the intervention. The access of endovascular treatment was obtained in the right common femoral artery, and a $5 \mathrm{~F}$ sheath was placed antegrade. The angiogram showed a $4-\mathrm{cm}$ length occlusion of popliteal artery and the distal artery was filled by the collaterals (Figure 1). A $5 \mathrm{~F}$ vertebral catheter and 0.035 soft Terumo wire were used as the first attempt to cross the lesion, but it was failed because of the tough proximal occlusion. Under the guide of roadmap, the stiff end of the wire was used to drill a hole in the beginning of occlusion and after that the soft tip in combination with the catheter getting through the whole occlusion. Angiogram through the catheter indicated the distal popliteal artery and the triple branches were patent to the foot without any embolization. The vertebral catheter was changed by a $4 \mathrm{~F}$ thrombolytic catheter with $20 \mathrm{~cm}$ work distance through the lesion and a 300,000 urokinase bolus was injected immediately. Then the urokinase was infused through the thrombolytic catheter by the concentration of $50,000 \mathrm{u}$ in $50 \mathrm{ml}$ saline per hour continuously. The coagulation time surveillance was performed every 8 hours. The first 24 hours angiogram didn't show any development at all, and a $5-\mathrm{mmX} 2-\mathrm{cm}$ balloon was used to enlarge the hole of the old thrombosis to make the blood flow through and enhance the catheter-directed thrombolysis activation

*Corresponding author: Chunshui He, Department of vascular Surgery, University Hospital of Chengdu University of Traditional Chinese Medicine, No. 39 Shier Qiao Road, Chengdu 610071, China, Tel: 86-18981885601; E-mail: chunshuihe@msn.com

Received September 16, 2013; Accepted September 28, 2013; Published September 30, 2013

Citation: He C, Hu Y, Huang Y, Fan X, Liu Y, et al. (2013) The Reconstruction of Popliteal Artery Injury after Knee Dislocation by Catheter-Directed Thrombolysis. J Vasc Med Surg 1: 115 doi: 10.4172/2329-6925.1000115

Copyright: @ $2013 \mathrm{He} \mathrm{C}$, et al. This is an open-access article distributed unde the terms of the Creative Commons Attribution License, which permits unrestricted use, distribution, and reproduction in any medium, provided the original author and source are credited. 


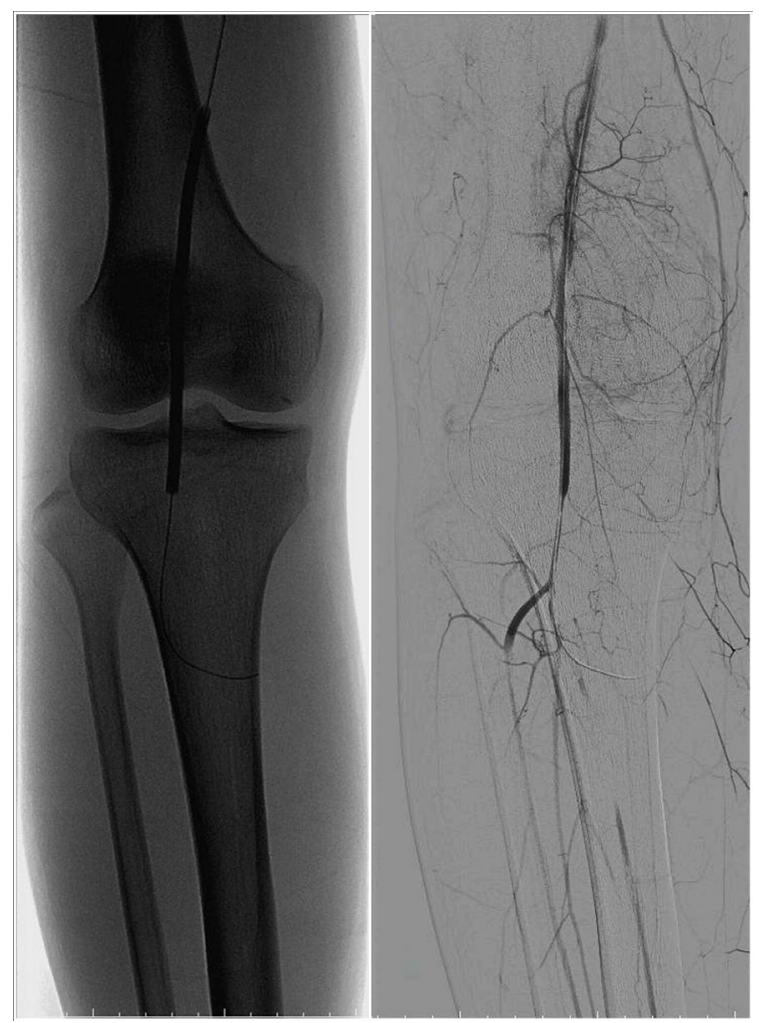

Figure 2: Balloon expansion 24 hours later and made a space through the lesion.

(Figure 2). After another 48 hours and 3000,000 units of urokinase in total infusion, the last angiography indicated the thrombosis was dissolved with a little stenosis residue in the patent popliteal artery with good runoff and no distal embolization (Figure 3). Aspirin $100 \mathrm{mg}$ and warfarin $3.75-5 \mathrm{mg}$ were prescribed daily thereafter to maintain the INR from 2-2.5. The pulse of dorsal pedal artery and posterior tibial artery could be palpated after the thrombolytic catheter withdrawal, and the ABI test showed the right was 0.86 and the left was 1.04. Ultrasound scan surveillance at 1 month later (before the reconstruction of knee ligaments), and 3 months later demonstrated patent popliteal artery and the $\mathrm{ABI}$ test indicated that the right is 0.93 and the left is 0.98 with palpable foot artery.

\section{Discussion}

Open revascularization procedure is still the standard treatment to the most cases of extremity vascular trauma. Disadvantages of an open surgery include difficult to expose and access arising from extremity edema, extensive incisions to control proximal and distal of the injured vessel, impairment of the vein and nerve nearby, and prolonged recovery after extensive operations with large incisions. An alternative approach is treatment with minimally invasive endovascular techniques since the endovascular approach may be the solution to avoid the zone of soft tissue injury while repairing the arterial injury. Recently, Endovascular treatment of extensive femoro-popliteal injury has been proposed to patients presenting with acute limb ischemia with favorable results comparing with open surgical revascularizations as far as limb salvage is concerned [5]. During the endovascular procedures in treating the popliteal artery injury after knee dislocation, the stents have been used to reconstruct the popliteal artery in most cases while remaining the possibility of stent fracture and restenosis [4]. Considering the mechanism of popliteal artery injury after knee dislocation, we want to revascularize the popliteal artery by catheter-directed thrombolysis without any stent left. Anatomically, the popliteal artery is tightly bound to the posterior aspect of the tibia while passing behind the fibrous arch of the soleus muscle. When the dislocation injury occurs, the linear distance across the popliteal space acutely increases and stretch injury occurs to the vessels. Direct contusion of the vessels by the adjacent bone is also possible during the injury process and is presumed to be most likely to occur in posterior knee dislocations. The arterial wall consists of 3 layers: intima, media and adventitia, which differ in their elastic properties. The single cell layer of the intima is inelastic and requires relatively little linear stretching to disrupt its confluent monolayer structure. This exposes the subendothelial layer, which naturally activates its procoagulant properties and causes acute thrombosis. The media consists of multilayered smooth muscle cell and elastic fiber zone and has modest resistance to stretch injury. The adventitia is the most resistant to stretch injury of the 3 layers of the arterial wall. It is often the only layer remaining intact after the injury event and maintains vascular integrity in most cases [1]. The pathology of the resected traumatic popliteal artery segment by open surgery we did before confirmed the main pathological change is the disruption of endothelial layer and media and the acute thrombosis development. Hutto and Reed [6] first reported the endovascular successful management of an acute blunt popliteal artery injury by extracting the thrombus under the distal embolization protection and performing a balloon angioplasty to the intimal lesion, but it is still possible the development of new thrombosis in situ since the existed intima and media trauma. Our theory behind using the catheter-directed thrombolysis is after dissolving the old thrombosis and maintaining focal thrombolytic activity continuously for one or two more days to get the endothelial recovery before new thrombosis development.

In this case, the most difficult step of the whole endovascular treatment is to get through the traumatic popliteal artery segment

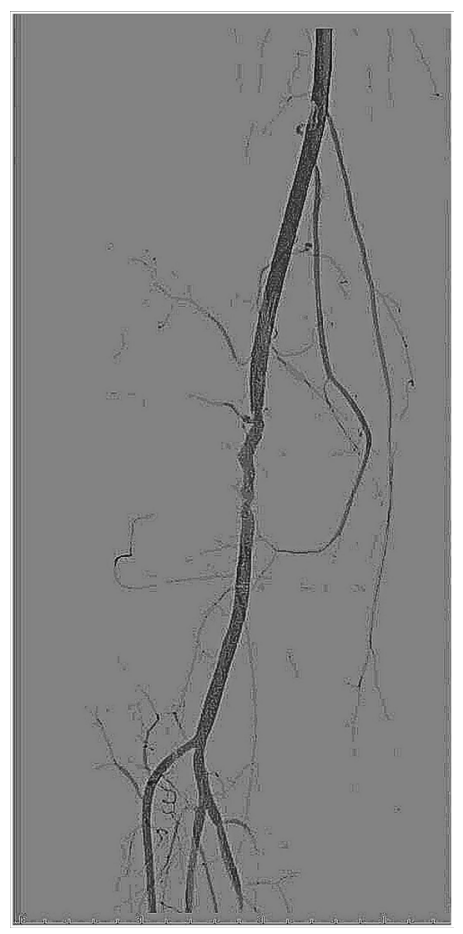

Figure 3: Completion angiogram. 
Citation: He C, Hu Y, Huang Y, Fan X, Liu Y, et al. (2013) The Reconstruction of Popliteal Artery Injury after Knee Dislocation by Catheter-Directed Thrombolysis. J Vasc Med Surg 1: 115 doi: 10.4172/2329-6925.1000115

Page 3 of 3

first, and we used the stiff end of the wire because usually this part of popliteal artery is straight. It is assumed that in the fresh injury case which is easier to get through the lesion and the time of thrombosis dissolution is shorter.

In conclusion, our approach is the first documented literature in which an endovascular repair of a blunt popliteal artery injury was performed successfully by catheter-directed thrombolysis in a subacutely ischemic extremity. In addition, the steps of endovascular treatment of popliteal artery injury after knee dislocation, especially for young patient, may be catheter-directed thrombolysis first, if there is any penetration of popliteal artery, then transfer to cover stent; if there is any stenosis residue, the drug eluting balloon or bare stent suitable for joint placement should be considered as a bailout.

\section{References}

1. Gray JL, Cindric M (2011) Management of arterial and venous injuries in the dislocated knee. Sports Med Arthrosc Rev 19: 131-138.

2. Frykberg E (2002) Popliteal vascular injuries. Surg Clin N Am 82: 67-89.

3. Zimmerman P, d'Audiffret A, Pillai L (2009) Endovascular repair of blunt extremity arterial injury: case report. Vasc and Endovasc Surg 43: 211-214.

4. Angiletta D, Impedovo G, Pestrichella F, Marotta V, Perilli F, et al. (2006) Blunt femoropopliteal trauma in a child: Is stent a good option. J Vasc Surg 44: 201205.

5. Alimi Y, Hakam Z, Hartung O, Boufi M, Barthelemy P, et al. (2008) Efficacy of viabahn in the treatment of severe superficial femoral artery lesions: which factors influence long-term patency? Eur J Endovasc Surg 33: 346-352.

6. Hutto J, Reed AB (2007) Endovascular repair of an acute blunt popliteal artery injury. J Vasc Surg 45: 188-190. 